\title{
Post-processing of time-sequences acquired during impact tests with the aid of a reference area
}

\author{
by S. Boccardi, G. M. Carlomagno, C. Meola
}

\begin{abstract}
Department of Industrial Engineering - Aerospace Division, University of Naples Federico II, Naples, Italy, simone.boccardi@unina.it, carmagno@unina.it,carmeola@unina.it
\end{abstract}

\begin{abstract}
The aim of this paper is concerned with post-processing of thermal images, acquired during impact tests of composite materials, with the aid of a reference unloaded specimen. The use of a reference area was already proposed by the authors to eliminate the detector noise while measuring small temperature variations. As a next step, now the use of a specimen identical to the loaded one (same material and surface finishing), is proposed as a reference for evaluating temperature variations, which can be exploited for assessing the extension of the impact damaged zone as well as of the overall impact affected area.
\end{abstract}

\section{Introduction}

Infrared thermography (IRT), thanks to its non-contact character and its multifarious applications, is gaining increasing attention from both the industrial and academic communities. This is supported from being the topic of the four International Symposia (QIRT, Thermosense, AITA, InfraMation) which continue to be held for many years. In particular, IRT has proved usefulness for monitoring the surface temperature changes, linked to thermo-elastic/plastic effects, which are experienced by a body when subjected to volume variations under load. This feature can be exploited to get information useful for assessing the performance of composites under impact. Indeed, composites are ever more dominating the aeronautical industry [1] and are asking for effective non-destructive evaluation techniques, as well for characterization techniques.

One main need for people involved in design and production of composites is for a fast and reliable evaluation of impact damage and extension of delamination caused by an impact of given energy $[2,3]$. There is common habit to impact the laminate at a given energy, evaluate, in non-destructive way, the induced damage extension and repeat the impact in another zone at increasing energy until the pre-set delamination has been reached. Of course, this operation is time consuming and, sometimes, not very accurate depending on the surface under exam and on the employed instrument. In fact, despite the many non-destructive testing (NDT) techniques today available, there is no certainty of having detected the actual extent of delamination. This because the two surfaces, in a delaminated zone, tend to adhere once the impactor moved away and the resulting discontinuity may be too small to affect the NDT signal.

As demonstrated by Meola and Carlomagno in previous work [4,5] an infrared imaging device can be advantageously exploited to monitor thermal effects evolving on the surface of composite materials when subjected to impact. This because the thermal signatures can be related to what occurred to the material under load and help understanding the impact damage mechanisms as well to estimate the overall impact affected zone.

While discriminating the presence of delamination, or fibres breakage, can be easily done owing to the local temperature rise, an accurate estimation of the effective extension of delamination is challenging. Some attempts were made $[4,6-8]$ but the problem is still not fully solved.

The aim of this work is to review the attempts previously made to evaluate the damaged area and to search for a more general procedure with the aid of a reference specimen [9]. In particular, the reference specimen may help to get rid of the instrument noise and of disturbances coming from the ambient $[10,11]$.

\section{Test setup and procedure}

Impact tests are performed with a modified Charpy pendulum which, as sketched in Fig. 1 allows the infrared camera to view the surface opposite to the impact. The specimen is positioned inside a specific lodge, which is made of two large plates, each having a window to allow for the contact with the hammer head from one side and optical view (by the infrared camera) from the other one [12]. A reference specimen is used for correction of the detector noise. More specifically, as shown in Fig.1, a smaller sample of the same material is attached over the specimen lodge so as to be included in the camera field of view without undergoing percussion. The impact energy is set by suitably adjusting the falling height of the hammer centre of gravity. The infrared camera acquires sequences of thermal images during the impact event. The used infrared camera is the SC6000 (Flir systems), which is equipped with a QWIP detector, working in the long wave (LW) 8-9 $\mu \mathrm{m}$ infrared band, NEDT < $35 \mathrm{mK}$, spatial resolution $640 \times 512$ pixels full frame, pixel size 25 $\mu \mathrm{m} \times 25 \mu \mathrm{m}$ and with a windowing option linked to frequency frame rate and temperature range. It is worth noting that the QWIP detector suffers from dark current noise, but is effective to sense also the feeble thermal radiation linked to very small temperature variations [10]. 


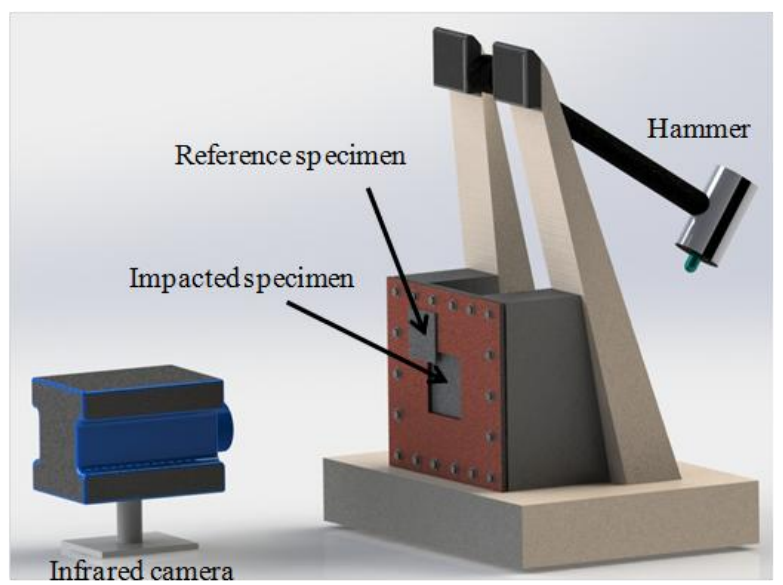

Fig. 1. Setup for impact tests with a modified Charpy pendulum

Any acquired thermal images sequence is post-processed to create a sequence of $\Delta T$ images accounting for temperature variations with respect to the initial unloaded condition:

$$
\Delta T(i, j, t)=T(i, j, t)-T(i, j, 0)
$$

$i$ and $j$ representing lines and columns of the surface temperature array, $t$ the time instant at which one image is recorded; more specifically, $t=0$ indicates the image of the sequence, before loading, for which the specimen surface is at ambient temperature.

\section{Evaluation of impact damaged area}

To locate the damage, it is important to discriminate between sound and damaged areas. This feature may seem simple to perform, but it represents a crucial task because of problems, linked to the different behaviours of materials under impact and to the instrument noise, which render difficult a generalization. In particular, thermoset matrix based composites become damaged on the side opposite to that struck by the impactor, often without any visible perception on the impacted side, or with only barely visible indentation [12]. Composites including a thermoplastic matrix behaves in a different manner under impact; in fact, they undergo visible deformation displaying an indentation (a small concavity) on the impacted side and a protrusion on the rear one [13]. Of course, these modifications are very small for very low impact energy, while they become ever more evident with increasing the impact energy. However, thermoplastic composites, while resembling ductile metals for the superficial appearance, are characterized by more complex impact damage mechanisms, which strongly depend on the type of matrix and on the impact energy.

Whatever the type of composite, it is important to know: if it is present a zone with material breakage and how wide it is, what happens to the material in absence of breakage, as well how wide is the overall impact-affected zone. Finding an answer to all of these questions may help design of composite materials and infrared thermography can be exploited to get such answers.

\subsection{A quick review of previous work}

Several attempts were made by the authors to assess the overall delamination from thermal images. The first one regarded glass/epoxy specimens impacted at different energies starting from very low values for which only thermoelastic effects were displayed without any permanent modification, up to energy high enough to cause fibres breakage [4]. The used criterion was to estimate the damaged area by encircling the appearing warm area. The latter was chosen by considering its time evolution (Fig. 11 of Ref 4 ) and the visible stain over the specimen surface which being translucent helped to see the occurred damage. This method supplied the real dimension of the occurred damage, but it is material dependent and cannot be assumed as general criterion.

Recently, a new procedure, named reference-based method, was proposed [7] and, later better conceived [8] to quantitatively evaluate the extension of the hot stain produced by the impact on thermoplastic composites. With this procedure, a sequence of $\Delta \mathrm{T}$ images is transformed into a sequence of binary images with any pixel forced to assume values equal to either 0 , or 1 , depending on a threshold value. Such a threshold value $\Delta \mathrm{T}_{\mathrm{T}}$, which is evaluated on a reference thermal image before the impact, is intended to account for the signal standard deviation of the used infrared camera and helps to discriminate temperature variations induced by the impact event.

Bearing in mind that we are analyzing thermal images, what we can measure is the extension of the warmed up area, which also means delineating the zone interested by temperature increase induced by the dissipated impact energy. Generally, from thermal images two zones may be recognized. The first one, depending on the impact energy, 


\subsection{1/qirt.2016.010}

may be small, sometimes including only slight hot spots, or wider including breakage and important delamination. The second one is quite large enclosing thin delaminations and the entire impact-affected area [6].

To ascertain the extension of each zone, it is important to set a boundary between sound and damaged zones, more specifically, a temperature difference threshold $\Delta T_{T}$ to be considered as a limit in the $\Delta T$ map to clearly identify a zone of a certain damage level. In particular, a high $\Delta T_{T}$ value surrounds a zone of important damage, while a lower $\Delta T_{T}$ value encircles a zone of lighter damage. For $\Delta \mathrm{T}_{\mathrm{T}}$ becoming very small the overall impact affected zone will be contoured.

\subsection{Limitations of previous method and new improvements}

One main limitation of the previously described reference-based method [8] is that it is referred to two average $\Delta T$ images (above a set of $\Delta T$ images) taken before and after impact, which cannot account for the random noise instrument. In particular, the warm $\Delta T_{\mathrm{W}}$ image, which is obtained as average amongst $50 \Delta \mathrm{T}$ images after impact is compared to the reference $\Delta T_{\mathrm{R}}$ image obtained as average amongst $100 \Delta \mathrm{T}$ images before impact. More specifically, a $\Delta T_{\mathrm{W}}$ image is transformed into a black/white binary image in which any pixel can assume a value equal to either 0 , or 1 , i.e. whether it is cold (black pixel), or warm (white pixel), respectively, according to:

$$
\left\{\begin{array}{l}
\Delta T_{w}(i, j)>\Delta T_{R}(i, j)+3 \sigma(\mathrm{i}, \mathrm{j}) \rightarrow \text { pixel }=1 \\
\Delta T_{w}(i, j)<\Delta T_{R}(i, j)+3 \sigma(\mathrm{i}, \mathrm{j}) \rightarrow \text { pixel }=0
\end{array}\right.
$$

The weak aspect of this method resides in the unpredictable random noise which may entail dummy shift of either the $\Delta T_{\mathrm{W}}$ image, or the $\Delta T_{\mathrm{R}}$ one making the two images no longer comparable. The noise instrument is time dependent and can be removed only by referring to an unloaded specimen enclosed in the camera field of view. On the other hand, the noise instrument affects any small $\Delta T$ value and then those at the boundary between delamination and sound material. Indeed, the signal recorded at such a boundary region may benefit from correction with the signal recorded in a sound zone [14]. Then, the reference-based method [8] is herein modified with the introduction of the reference unloaded specimen and is named noise correction reference based method and abbreviated as NCR method. This means that the $\Delta T$ images coming from Eq. (1) are restored by comparison to the average $\Delta T_{R N}$ signal recorded at the same time over the reference specimen and then a $\Delta T_{\mathrm{C}}$ :

$$
\Delta T_{C}(i, j, t)=\Delta T(i, j, t)-\Delta T_{R N}(t)
$$

sequence of images is created, which is used for implementation of the reference-based method. The substantial difference between the two methods consists in the corrected $\Delta T_{C}$ sequence to be used instead of the uncorrected $\Delta T$ one, or better in the addition of Eq. (3) in the post-processing procedure.

Another feature worth of attention regards the number of images post impact to be included in the average $\Delta T_{\mathrm{W}}$ image. In fact, in Ref. [8] the number of $50 \Delta T$ images has been considered, also owing to the specific materials under consideration, to be enough to account for the development of impact damaging related heat transfer mechanisms. In reality, this is a crucial aspect of great concern. In the light of the development of a general method to be applied with whatever material, it is important to, first of all, clear the meaning of the $\Delta T_{\mathrm{W}}$ image. While the $\Delta T_{\mathrm{R}}$ image simply represents the conditions before starting of the impact and it can be either chosen as a single image, or the average amongst a certain number of images without any physical implication, the choice of the $\Delta T_{\mathrm{W}}$ image is more critical and may affect the final result. This implies the set of $\Delta T$ images used to calculate $\Delta T_{\mathrm{W}}$ to be chosen by considering the material thermal diffusion time and its damaging way. It has to be considered that in thick parts the damage spreads out from the top (impact side) to the bottom through the typical pine tree configuration [3]. Conversely, for thin laminates the damage starts from the rear surface (opposite to the impact) and evolves in the reversed pine tree pattern.

A specific case study is herein considered involving a thermoset matrix (epoxy resin) reinforced with carbon fibres, which belong to the class of composites, which are largely employed in aircraft construction. The used specimens are thin and then the impact damage starts on the side viewed by the infrared camera (i.e., side opposite to that struck by the impactor). The estimation of the damaged area is performed in two ways: by applying first the reference-based method of Ref [8] and then the NCR method with $\Delta T_{W}=50 \Delta T$ images. The obtained results are compared to try to quantify the errors induced by the noise instrument for a decision whether the noise has to be accounted for, or may be neglected. However, it is worth noting that, due to the random nature of the instrument noise, there may be the lucky case of absence of jumps, turning to a quasi perfect overlap of results.

\section{Case study}

Two carbon/epoxy specimens are considered and impacted with the modified Charpy pendulum (Fig. 1). One named CFRP-U includes unidirectional fibres, which are stacked according to the sequence $(0,45 .-45.90)_{s}$ to reach a total thickness of $2.4 \mathrm{~mm}$ and is impacted at $E=8 \mathrm{~J}$. The other one named CFRP-F includes woven fabric layers to have a thickness of about $1.5 \mathrm{~mm}$ and is impacted at $E=2.8 \mathrm{~J}$. Some $\Delta T$ images of the two specimens, extracted from the $\Delta T$ sequence created with Eq. 1, are shown in Figs. 2 and 3. 

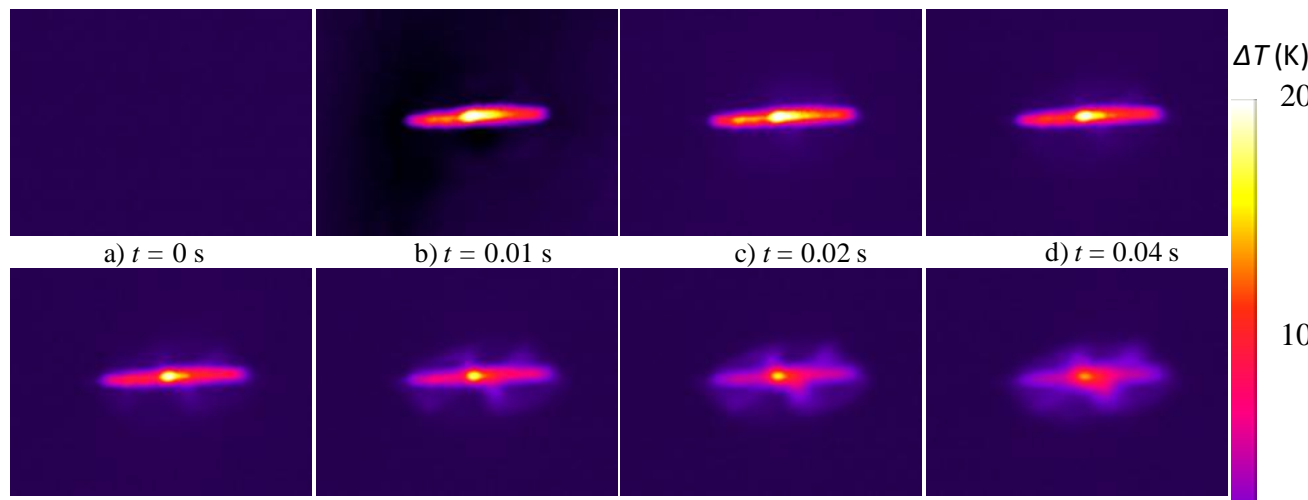

c) $t=0.02 \mathrm{~s}$

d) $t=0.04$
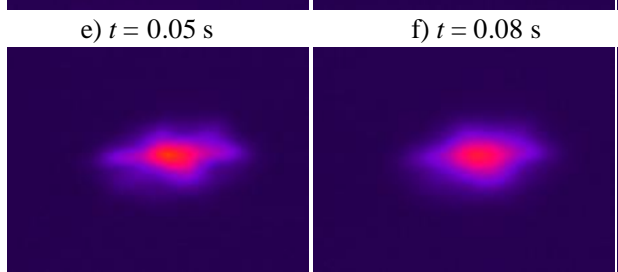

j) $t=0.84 \mathrm{~s}$
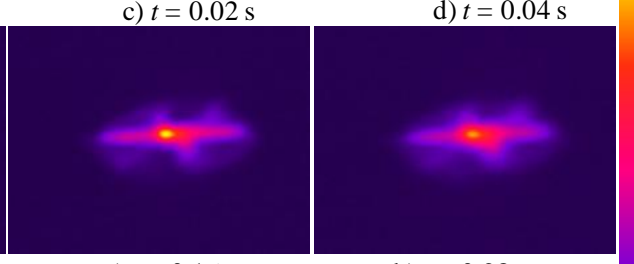

i) $t=0.48 \mathrm{~s}$

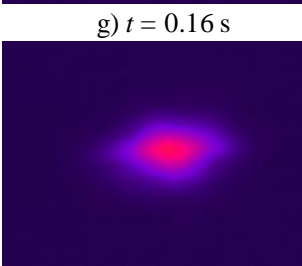

k) $t=1.10 \mathrm{~s}$ h) $t=0.28 \mathrm{~s}$

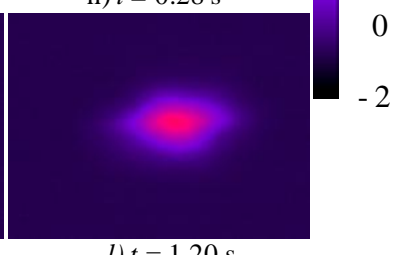

l) $t=1.20 \mathrm{~s}$

Fig. 2. Some $\Delta T$ images of the $C F R P-U$ specimen impacted at $E=8 \mathrm{~J}$

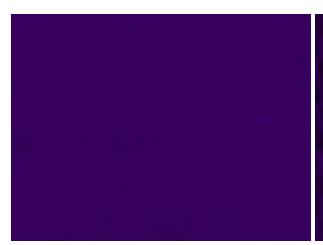

a) $t=0 \mathrm{~s}$

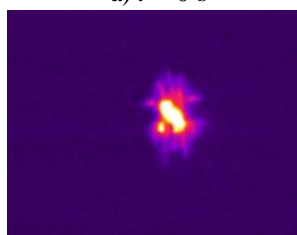

e) $t=0.05 \mathrm{~s}$

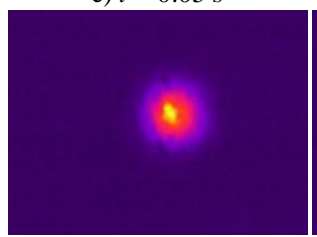

i) $t=0.89 \mathrm{~s}$

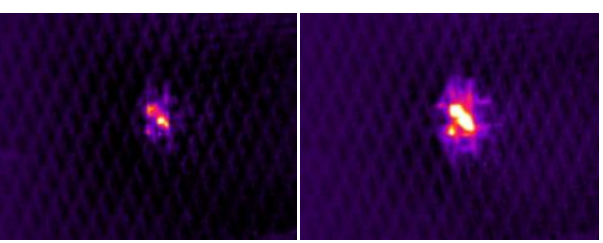

b) $t=0.01 \mathrm{~s}$

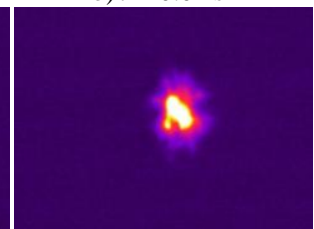

f) $t=0.10 \mathrm{~s}$

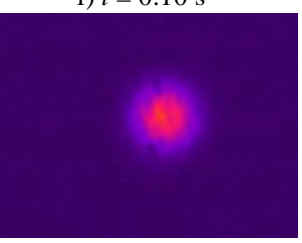

j) $t=1.50 \mathrm{~s}$

c) $t=0.02 \mathrm{~s}$

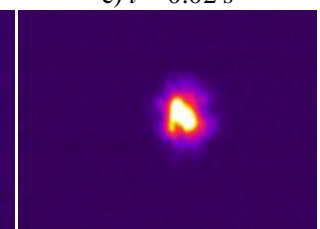

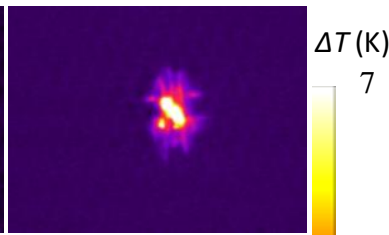

d) $t=0.04 \mathrm{~s}$

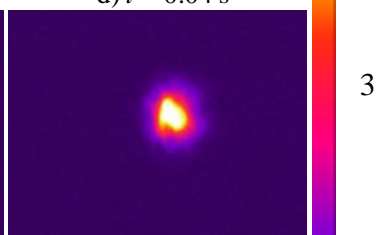

h) $t=0.29 \mathrm{~s}$

Fig. 3. Some $\triangle T$ images of the CFRP-F specimen impacted at $E=2.8 \mathrm{~J}$

As it can be seen, the first image is of almost uniform colour referring to $\Delta T=0$ before the impact. Sudden at the impact, there is a cooling down in the peripheral zones, due to the surface bending under the pushing impactor force, and heating up at the center, due to dissipation of impact energy [4-8]. Information on the material behaviour under impact can be derived from the evolution with time of thermal signatures. The attention of the present work is mostly towards the estimation of the damaged area, which is intended to coincide with the warm area and then the scale is optimized to see heating effects by scarifying negative values (thermo-elastic effects).

Looking at the $\Delta T$ images, the most important thing that catches eyes for the CFRP-U specimen is the oblong hot stripe, which is characterized by high temperature rise over $20 \mathrm{~K}$ for $t=0.01 \mathrm{~s}$ till $t=0.04 \mathrm{~s}$ (Figs. $2 \mathrm{~b}-\mathrm{d}$ ); this indicates breakage of fibres along their external horizontal direction. Conversely, the specimen CFRP-F is characterized by a hot zone which appears as a small oblong stripe along fibres at $-45^{\circ}$ (Fig. $3 \mathrm{~b}, \mathrm{c}$ ) and then enlarges with the damage evolving along the woven fabric directions, becoming almost circular at $t=0.29 \mathrm{~s}$ (Fig. 3h). For both specimens, the fibres breakage starts on the surface viewed by the infrared camera, i.e., that opposite to the impacted one. Going on in time, the $\Delta T$ peak weakens and let to distinguish the overall heat affected area around. Finding the border of such heat affected zone would mean locate the extension of delamination. 


\subsection{1/qirt.2016.010}

\subsection{Evaluation of damage extension from thermal images}

The damage extension is appraised from the extension of the warm area. Then, $\Delta T$ images are post-processed by considering first the reference-based method [8] and then the newly NCR implemented method with noise correction; for both methods $\Delta T_{\mathrm{R}}=100 \Delta T$ images and $\Delta T_{\mathrm{W}}=50 \Delta T$ images. The contoured maps for the two specimens are shown in Figs. 4 and 5. At first glance, it seems the two areas of Fig. 4 look identical, while the area of Fig. $5 \mathrm{~b}$ is greater than that of Fig. 5a; this may let to suppose absence of noise in the first case. To better clarify this aspect, the $\Delta T$ distribution in time in the three zones A1, A2 and A3 are shown in Figs. $6 a$ and $7 a$ for the two specimens; in particular, each zone is composed of $3 \times 3$ pixels. As it can be seen only plots relative to the CFRP-F specimen display one jump before the impact and another one after the impact; the latter is more pronounced for smaller $\Delta T$ values (point more distant form the hottest central zone). Then, the $\Delta T$ plots in $\mathrm{A} 3$ are shown again with amplified scale in Fig. $6 \mathrm{~b}$ and $7 \mathrm{~b}$ for both specimens. In addition, the corrected $\Delta T_{C}$ plots are shown in Figs. $6 \mathrm{c}$ and $7 \mathrm{c}$. As displayed by the histograms reported in Figs. 8 and 9, also in the absence of visible jumps some noise is always present and it is reduced by applying Eq. (3). It is worth noting that each histogram includes the 100 images (before impact) used to obtain the average $\Delta T_{R}$ image.

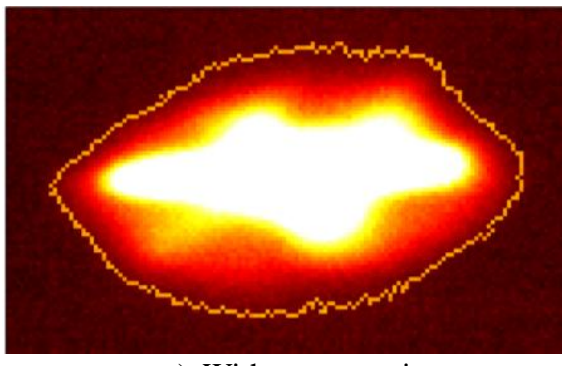

a) Without correction

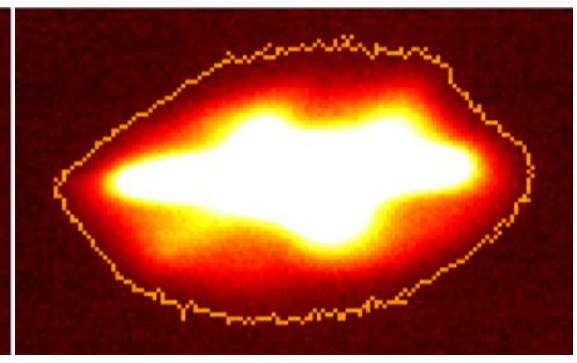

b) With correction

Fig. 4. Contoured maps of CFRP-U specimen, $E=8 \mathrm{~J}$

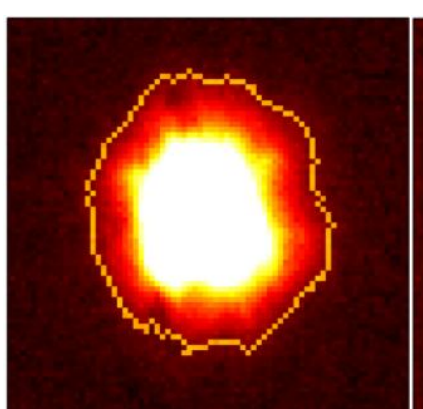

a) Without correction

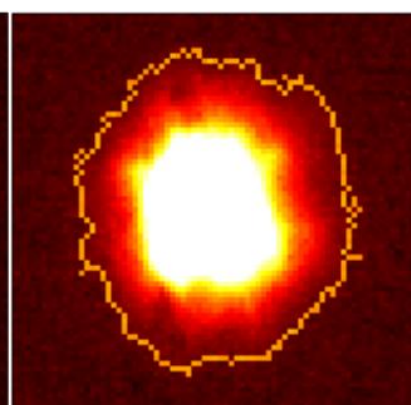

b) With correction

Fig. 5. Contoured maps of CFRP-F specimen, $E=2.8 \mathrm{~J}$

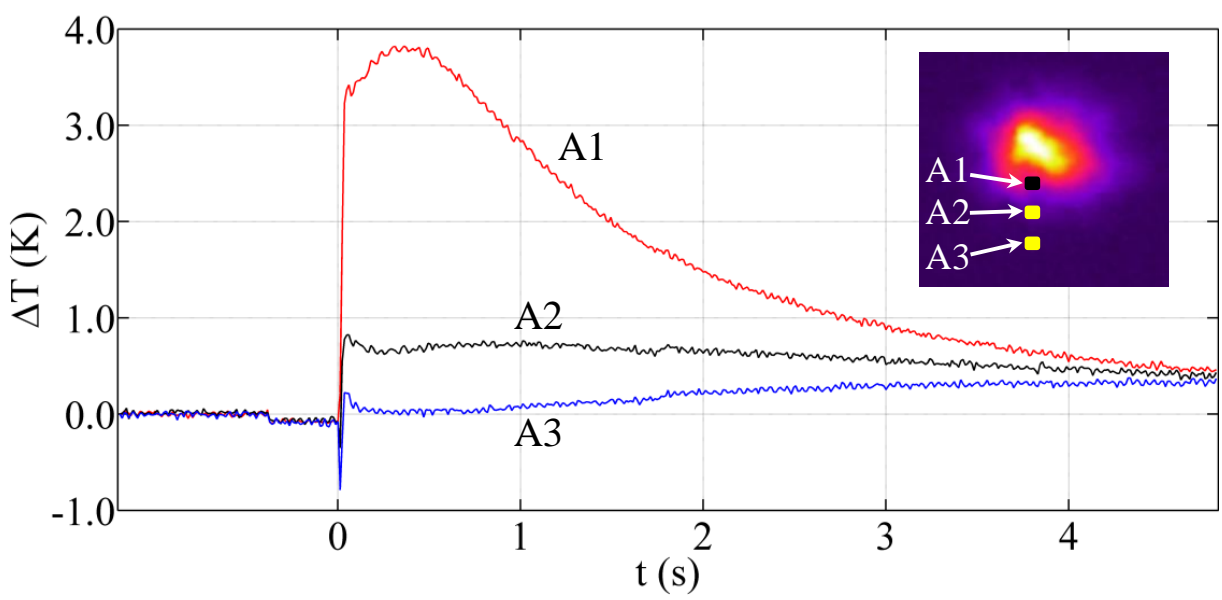

a) Plots in three points 


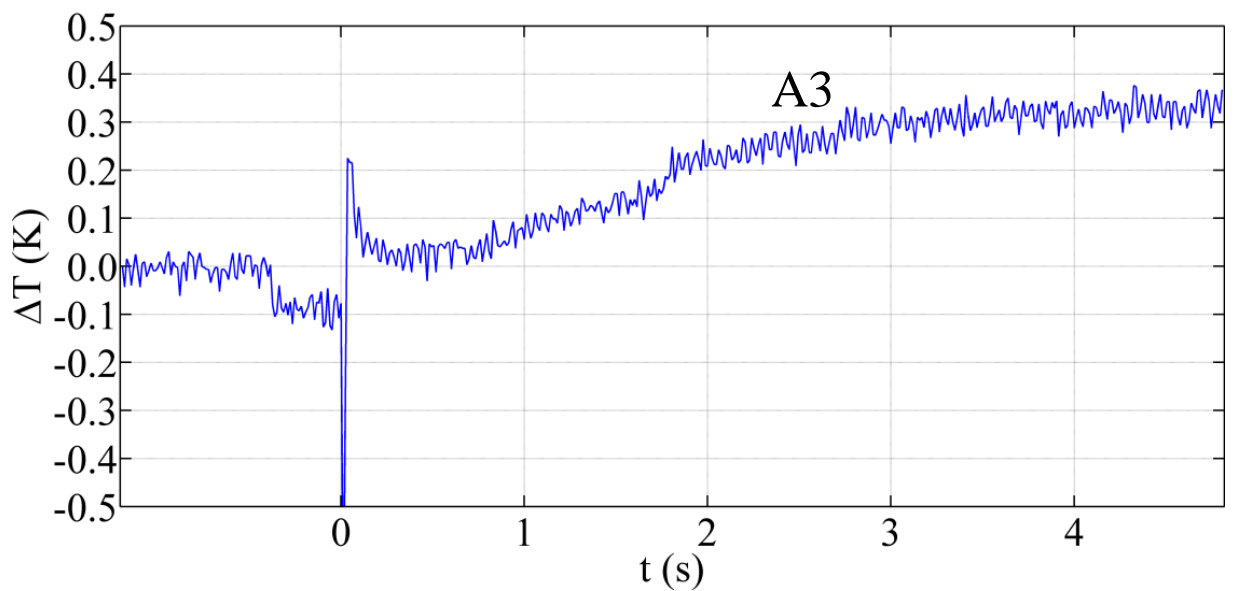

b) Uncorrected $\Delta T$ plot in A3 with amplified scale

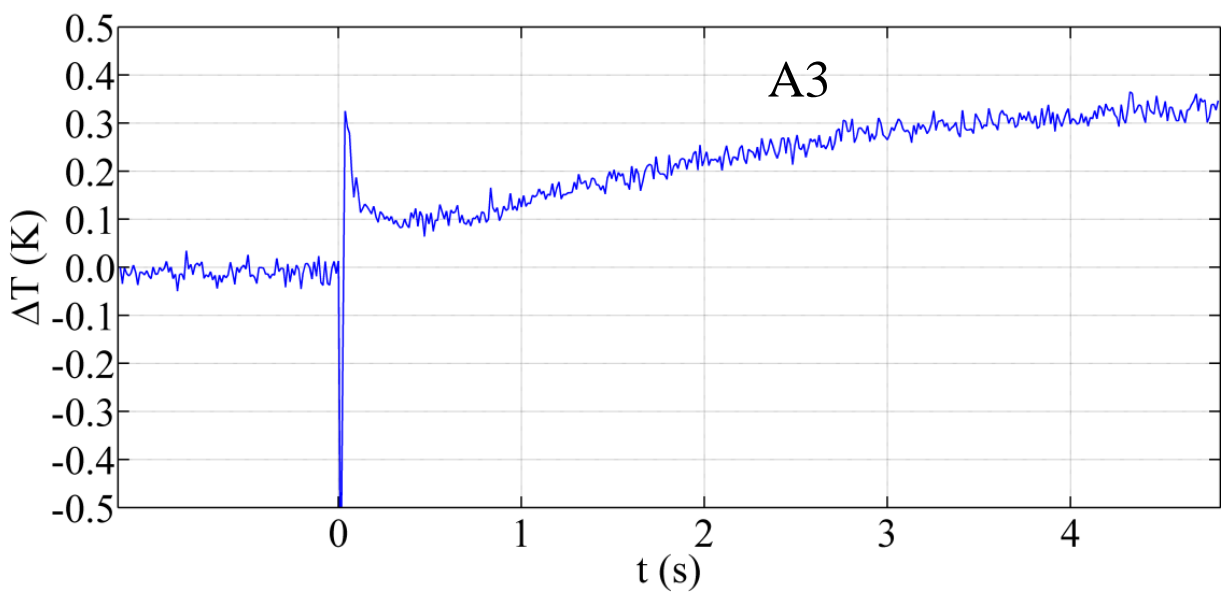

c) Corrected $\Delta T_{C}$ plot in $A 3$ with amplified scale

Fig. 6. Some $\Delta T$ plots of CFRP-F specimen, $E=2.8 \mathrm{~J}$

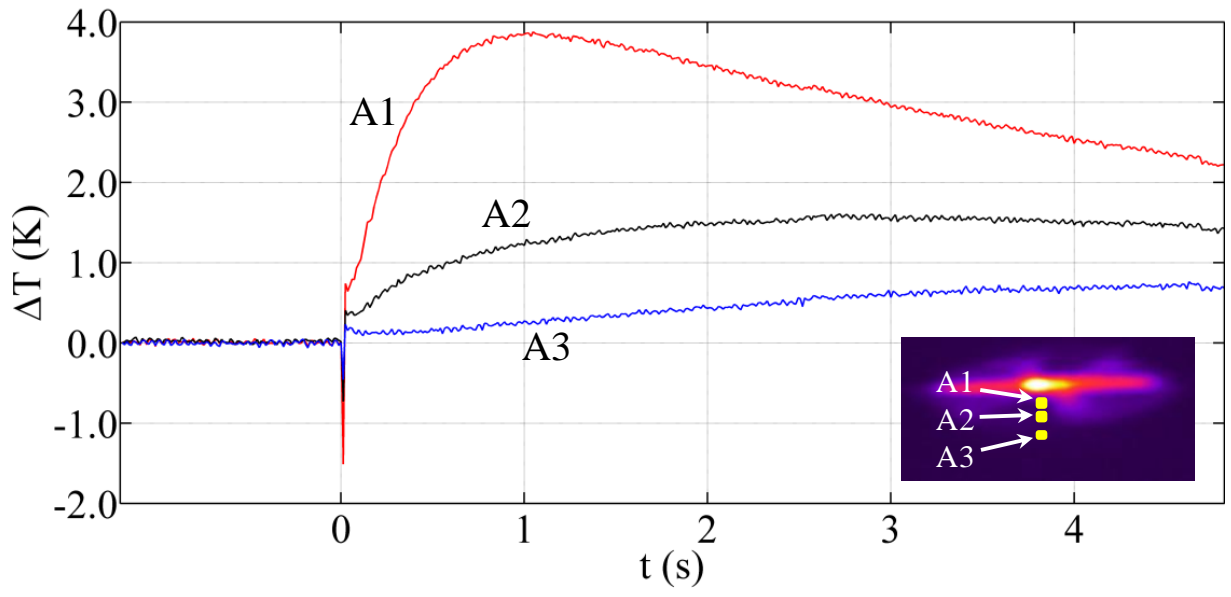

a) Plots in three points 


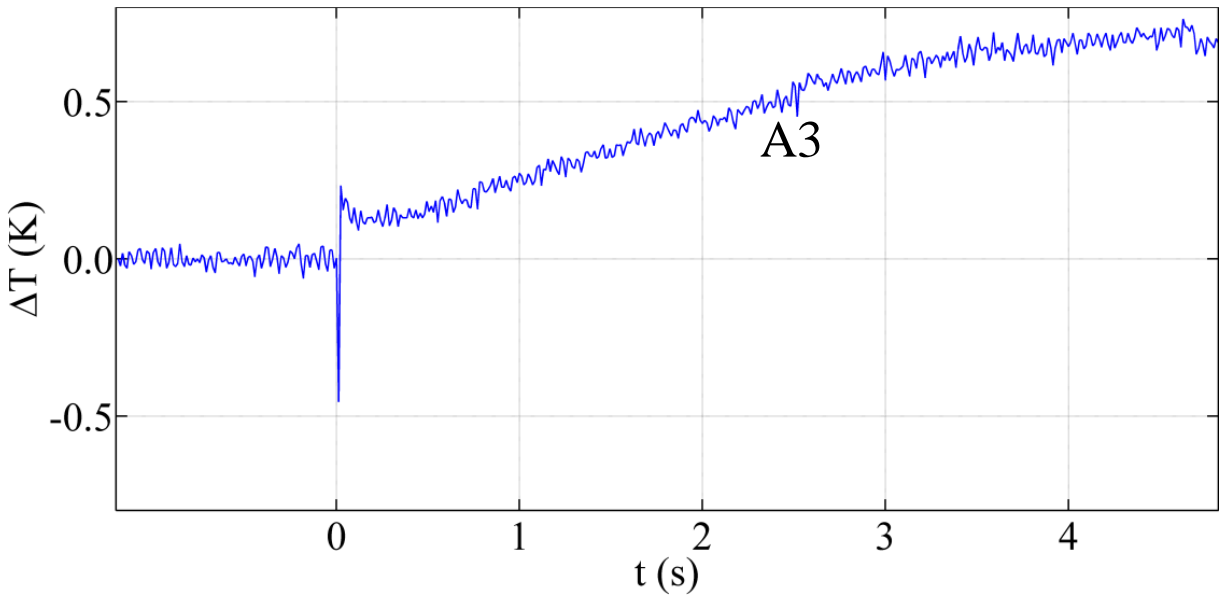

b) Uncorrected $\Delta T$ plot in A3 with amplified scale

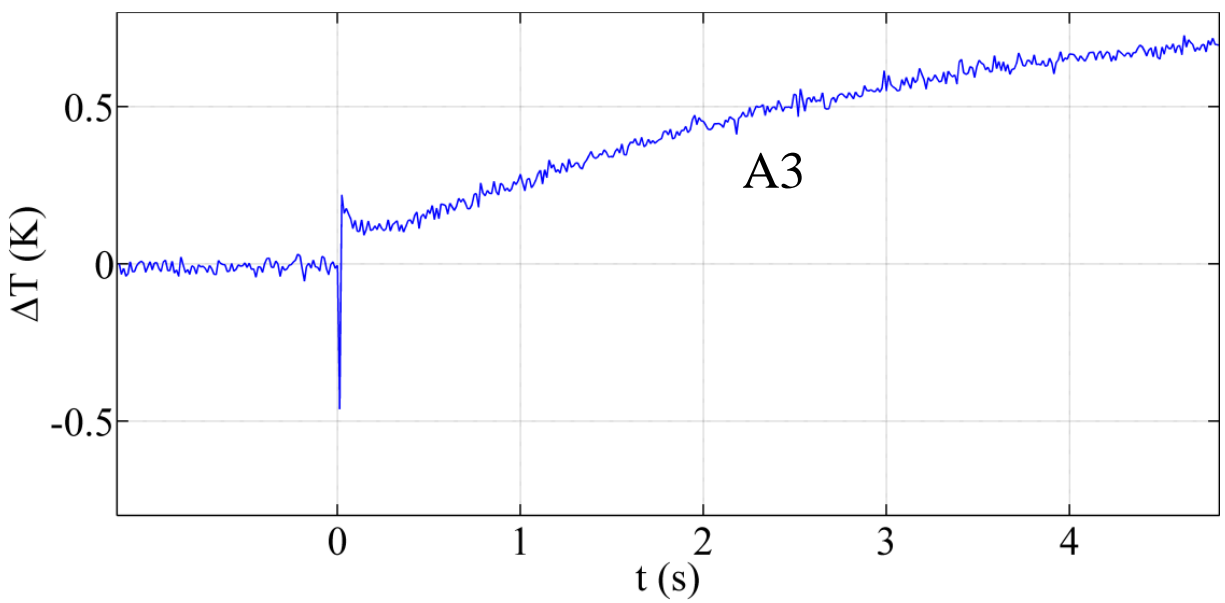

c) Corrected $\Delta T_{C}$ plot in $A 3$ with amplified scale

Fig. 7. Some $\Delta T$ plots of CFRP-U specimen, $E=8 \mathrm{~J}$

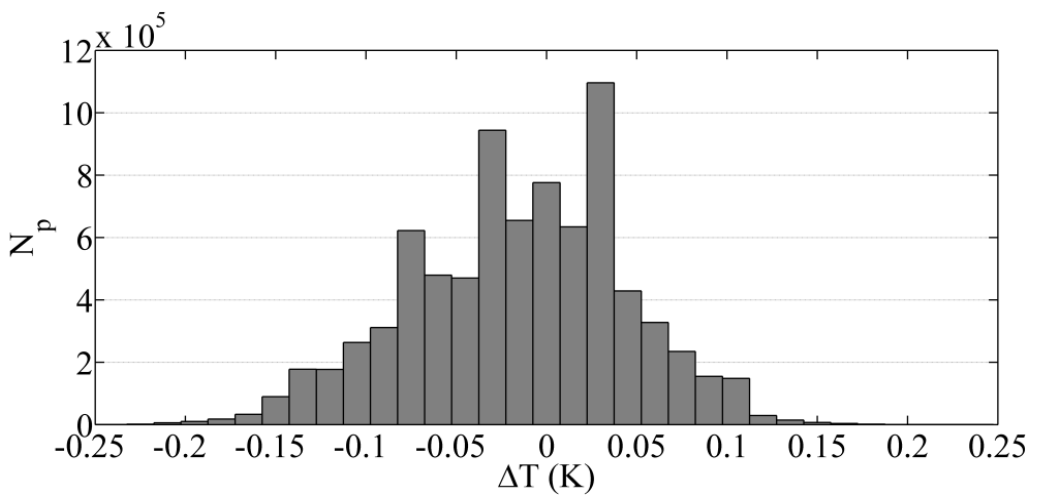

a) Uncorrected 


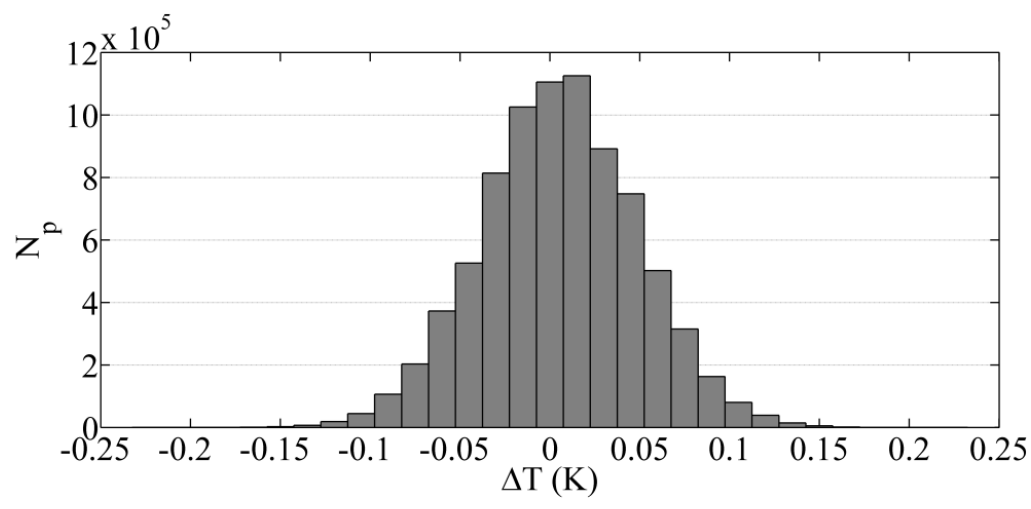

b) Corrected

Fig. 8. Histogram of the $100 \Delta T$ images before impact of CFRP-F specimen, $E=2.8 \mathrm{~J}$

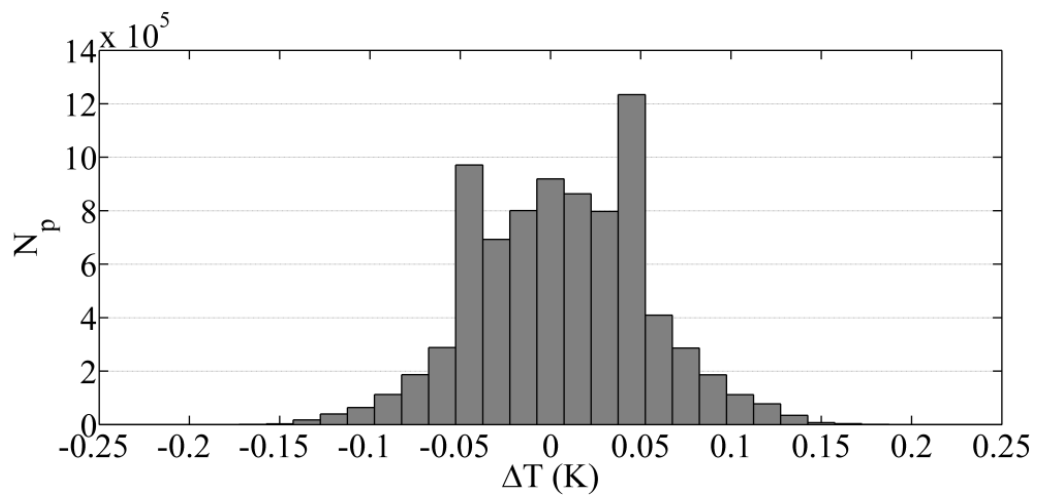

a) Uncorrected

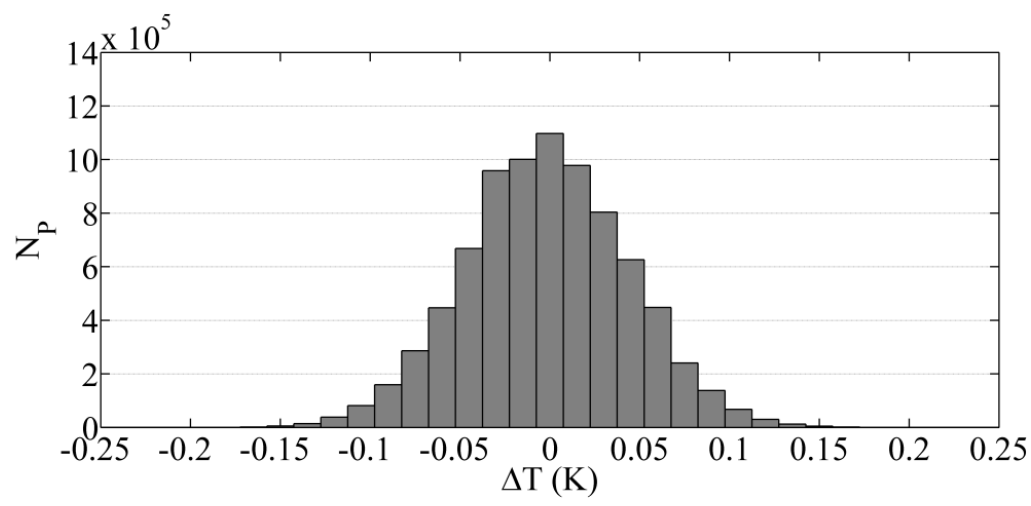

b) Corrected

Fig. 9. Histogram of the $100 \Delta T$ images before impact of CFRP-U specimen, $E=8 \mathrm{~J}$

The evaluation of the warm area $A_{h}$ is obtained by counting the number of hot pixels $P_{h}$ enclosed and considering the camera spatial resolution $s_{r}$, which means applying the relationship:

$$
A_{h}=P_{h} * s_{r}^{2}
$$

In particular, two $A_{h}$ values are obtained owing to the two data reduction methods with and without noise corrections; the corrected values are named $A_{h c}$. The two $A_{h}$ and $A_{h c}$ values with maxima $\left(\Delta T_{M a x}\right)$ and minima $\left(\Delta T_{\text {Min }}\right) \Delta T$ values and other specimens as well test details are collected in Table 1. The most important finding regards the $A_{h c}$ value which is greater than the uncorrected one for both specimens; of course, this difference increases with increasing the noise level and mostly with the presence of jumps. 
Table 1. Some details and results of used specimens

\begin{tabular}{|c|c|c|c|c|c|c|}
\hline Code & $\begin{array}{c}\text { Thickness } \\
(\mathrm{mm})\end{array}$ & $\mathrm{E}(\mathrm{J})$ & $\Delta \mathrm{T}_{\text {Max }}(\mathrm{K})$ & $\Delta \mathrm{T}_{\text {Min }}(\mathrm{K})$ & $\begin{array}{c}A_{h}\left(\mathrm{~mm}^{2}\right) \\
{[\mathrm{Ref} .8]}\end{array}$ & $\begin{array}{c}A_{h C}\left(\mathrm{~mm}^{2}\right) \\
{[\mathrm{NCR}]}\end{array}$ \\
\hline CFRP-U & 2.4 & 8 & 24.5 & -1.6 & 351 & 361 \\
\hline CFRP-F & 1.5 & 2.8 & 17 & -2.8 & 117 & 152 \\
\hline
\end{tabular}

\section{Conclusion}

The intention of this work was to find a general criterion for the evaluation of the extension of the impact damaged area of composite materials starting from a sequence of thermal images recorded during the impact event. For the purpose a previously proposed method [8] has been revisited and improved by accounting of the instrument and environmental noise through the use of a reference unloaded specimen. The used camera was the SC6000 equipped with the QWIP detector, which suffers from dark current noise. The obtained results show that the new NCR method is effective in eliminating the random jumps, which may affect the recorded signal, and in consistently reducing other types of noise. This correction makes possible to resolve the small temperature variations at the boundary of the warm area representative of the overall extension of delamination.

As an important observation, the possibility to evaluate the overall extension of the impact damage from thermographic images has a double value. On one side, offers to composite materials developer a fast and accurate means to assess the impact performance of new materials. On the other side opens the door to a wider exploitation of infrared thermography.

However, it has to be noted that many other tests involving composites of different type and thickness as well tests at different impact energy are necessary for the validation of the method. Of course, with a wide set of materials, the construction of the $\Delta T_{W}$, which in the present work has been set equal to $50 \Delta T$ images, requires more attention by considering the characteristic impact damage configuration (pine tree, reverse pine tree, or something else) and the characteristic thermal diffusion time with related heat transfer considerations.

\section{REFERENCES}

[1] Soutis C., "Fiber reinforced composite in aircraft construction". Progr Aerosp Sci, vol. 41, pp. 143-151, 2005.

[2] Richardson M.O.W., Wisheart M.J., "Review of low-velocity impact properties of composite materials". Composites Part A, vol. 27, pp. 1123-1131, 1996.

[3] Abrate S., (Editor) "Impact Engineering of Composite Structures", Springer Wien, New York, 2011.

[4] Meola C., Carlomagno G.M., "Impact damage in GFRP: new insights with Infrared Thermography". Composites Part A, vol.41, pp- 1839-1847, 2010.

[5] Meola C., Carlomagno G.M., "Infrared thermography to evaluate impact damage in glass/epoxy with manufacturing defects". Int. J. Impact Engineering, vol.67, pp. 1-11, 2014.

[6] Meola C., Boccardi S., Carlomagno G.M., "Infrared thermography in the evaluation of aerospace composite materials". Chapter 5, Woodhead Publishing in Materials, in press.

[7] Boccardi S., Carlomagno G.M., Meola C., Russo P., Simeoli G., "Monitoring impact damaging of thermoplastic composites", Journal of Physics: Conference Series 658 (2015) (XXII AIVELA Annual Meeting) 012005 doi:10.1088/1742-6596/658/1/012005.

[8] Boccardi S., Carlomagno G.M., Simeoli G., Russo P., Meola C., "Evaluation of impact affected areas of glass fibres thermoplastic composites from thermographic images", Measurement Science and Technology, to be published.

[9] Boccardi S., Carlomagno G.M., Bonavolontà C., Valentino M., Meola C., "Infrared thermography to monitor Glare ${ }^{\circledR}$ under cyclic bending tests with correction of camera noise" in Proc. QIRT 2014, Bordeaux, France, 7-11 July 2014

[10] Meola C., Boccardi S., Carlomagno G.M., "Measurements of very small temperature variations with LWIR QWIP infrared camera", Infrared Physics and Technology, vol. 72, pp. 195-203, 2015.

[11] Boccardi S., Carlomagno G.M., Meola C., "Remote Sensing of Temperature-Stress Coupled Effects", in Proc. IARIA ALL SENSORS 2016, Venezia, 24-28 April 2016.

[12] Meola C., Boccardi S., Carlomagno G.M., Boffa N.D., Monaco E., Ricci F., "Nondestructive evaluation of carbon fibre reinforced composites with infrared thermography and ultrasonics", Composite Structures, vol. 134, pp. 845-853, 2015.

[13] Boccardi S., Carlomagno G.M., Meola C., Russo P., Simeoli G., "Infrared thermography to evaluate impact damaging of thermoplastic composites", in Proc. QIRT 2014, Bordeaux, France, 7-11 July 2014. paper 214.

[14] Boccardi S., Carlomagno G.M., Meola C., "Measurements with a QWIP infrared camera: signal treatment and noise correction", in Proc. AITA 2015 Advanced Infrared Technology and Applications, Pisa, 29 September- 2 October 2015. 